\title{
Consenso Nacional de Enfermería sobre el manejo del niño con lesión cerebral por traumatismo de cráneo grave
}

\author{
National Nursing Consensus on management of severe traumatic \\ brain injury in children
}

\author{
Dr. José M. Palacio ${ }^{a}$ Lic. Karina Rojas ${ }^{a}$, Lic. Berta Balmaceda ${ }^{a}$, Lic. Adrián Bustamante ${ }^{b}$, \\ Lic. Vanesa Mogro ${ }^{a}$, Lic. Edith Cavallo ${ }^{a}$, Lic. Yanina Durán ${ }^{a}$, Lic. Cristina Ulloa ${ }^{a}$, \\ Lic. Pedro Villarreal ${ }^{a}$, Lic. Diana Roopel ${ }^{b}$, Dr. Roberto Jaimovichc, Dra. Eugenia Kenny ${ }^{d}$, \\ Dr. Edgardo Rodríguez ${ }^{d}$, Dr. Guillermo Moreno ${ }^{d}$, Dr. Martín Truszkowskid, \\ Dr. Tomás Iolsterd ${ }^{2}$ Dr. Germán Bonetto ${ }^{d}$ y Dra. Susana Ciruzzi ${ }^{e}$ \\ Colaboradores: Dr. Andrés Fessiaf, Dr. Miguel Grijalbac y Lic. Paola Díaz
}

\section{RESUMEN}

El profesional de enfermería que atiende a niños críticamente enfermos con lesión cerebral es un miembro clave dentro del equipo intensivista pediátrico, ya que, mediante la evaluación exhaustiva, planifica cuidados de enfermería de manera integral dirigidos al niño y a la familia. La enfermería como una profesión que entiende la salud de la persona humana desde una mirada integral cumple un rol esencial (indispensable) en el cuidado de los pacientes, principalmente, a nivel hospitalario (como domiciliario).

Por ende, el rol que juega la enfermería en la atención de un niño con traumatismo encéfalocraneano grave es crucial en la gestión del cuidado infantil.

Esta guía ofrece recomendaciones de enfermería sobre los cuidados neurocríticos focalizando una mirada sistémica basada en diagnósticos de enfermería según la Asociación Americana de Diagnósticos de Enfermería.

Palabras clave: cuidados críticos, lesión cerebral, procesos de enfermería, niño.

\section{ABSTRACT}

The nursing professional who treats critically ill children with cerebral injury is a key element within the pediatric intensive care team, since, through exhaustive assessment, plans nursing care in an integral manner aimed at the child and the family, and plays an essential role in the care of patients mainly at the hospital level (as well as at home).

Therefore, the role played by nursing in the care of children with severe brain trauma is crucial. This guide offers nursing recommendations on neurocritical care, focusing on a systemic view based on nursing diagnoses according to the North American Nursing Diagnosis Association. Key words: critical care, brain injury, nursing process, child.

http: / / dx.doi.org/10.5546/ aap.2019.S157

Cómo citar: Palacio JM, Rojas K, Balmaceda B, Bustamante A, et al. Consenso Nacional de Enfermería sobre el manejo del niño con lesión cerebral por traumatismo de cráneo grave. Arch Argent Pediatr

2019;117 Supl 4:S157-S174.

\section{CONTENIDO DE LA GUÍA}

- Introducción

- Fisiopatogenia

- Metodología

- Objetivos según el soporte vital pediátrico avanzado

- Planificación y organización de la Unidad de Cuidados Intensivos Pediátricos

- Recepción del niño con traumatismo encéfalo-craneano grave en la Unidad de Cuidados Intensivos Pediátricos

- Gestión del cuidado de acuerdo con los diagnósticos de enfermería, objetivos y acciones fundadas según patrones:

- Patrón neurológico

- Patrón hemodinámico

- Patrón respiratorio

- Patrón urinario

- Patrón metabólico y nutricional

- Higiene, confort y seguridad del niño

- Cuidado humanizado en la Unidad de Cuidados Intensivos Pediátricos

- Discusión

- Conclusiones

- Bibliografía

- Anexos
Conflicto de intereses: Ninguno que declarar.

Recibido: 2-11-2018 Aceptado: 21-1-2019 


\section{INTRODUCCIÓN}

La Academia Americana de Pediatría, en conjunto con la Organización Mundial de la Salud (OMS), definen que los accidentes son "la enfermedad por negligencia de la sociedad moderna" y continúan siendo la principal causa de muerte en la población pediátrica. Por ello, el Comité Nacional de Emergencias y Cuidados Críticos de la Sociedad Argentina de Pediatría (SAP), en concordancia con los Dres. B. Fuhrman y J. Zimmerman, ${ }^{1}$ define el traumatismo encéfalocraneano grave (TECG) como toda lesión primaria o secundaria del encéfalo y la cubierta ósea en un grupo vulnerable de pacientes menores de 18 años y que presenten un puntaje de Glasgow de entre 3 y 8 puntos.

Dentro de la epidemiología nacional, la incidencia del traumatismo encéfalo-craneano (TEC) admite que el $70 \%$ está asociada a accidentes en la vía pública; el $25 \%$, a caídas de sus propias alturas, relacionadas con su deambulación, y un $5 \%$, pero con gran impacto, lo constituye el síndrome del bebé sacudido (shaken baby syndrome), reflejado en las hemorragias intracerebrales.

Los avances de la tomografía axial computada (TAC) y el monitoreo de la presión de perfusión cerebral (PPC) fueron los "responsables, en los últimos 20 años," de posibilitar un tratamiento más objetivo y preciso, y las intervenciones preventivas fueron las más eficaces para disminuir la morbimortalidad. ${ }^{2}$

\section{FISIOPATOGENIA}

En el TECG, se involucran dos tipos de lesión:

La lesión primaria. Se produce en el momento del impacto, a consecuencia del traumatismo directo sobre el cerebro, o por las fuerzas de aceleración o desaceleración en la substancia blanca. Incluye la laceración y contusión cerebral y las disrupciones vasculares y neuronales. Una vez producidas estas lesiones, son difícilmente modificables por la intervención terapéutica.

La lesión secundaria. Resulta de los procesos intracraneales y sistémicos que acontecen como reacción a la lesión primaria y contribuyen al daño y a la muerte neuronal. A nivel intracraneal, pueden aparecer edema cerebral, hemorragias intracraneales (axiales o extraaxiales), convulsiones, etc., con un intervalo variable desde el traumatismo. A nivel sistémico, debido a la lesión cerebral primaria u otras lesiones asociadas, se pueden producir alteraciones que comprometen aún más la perfusión neuronal, como hipotensión arterial, hipoxemia, hipercapnia o anemia. El daño cerebral secundario, a diferencia del primario, es potencialmente tratable $\mathrm{y}$, en su mayoría, anticipable. ${ }^{3}$

La clasificación del TEC según la Escala de Coma Glasgow (Glasgow Coma Scale; GCS, por sus siglas en inglés) ha facilitado al profesional de salud evaluar de manera práctica y objetiva el nivel de estado de alerta en los seres humanos cuando son víctimas de un TEC y, en particular, a la población infantil. Esta fue creada en 1974 por Bryan Jennett y Graham Teasdale, miembros del Instituto de Ciencias Neurológicas de la Universidad de Glasgow (véase el Anexo 1).

La importancia de esta clasificación radica en que permite monitorizar la evolución del niño con traumatismo, intercambiar información entre los profesionales que lo atienden de una manera rápida, concisa, y orientar el enfoque inicial del niño, a la vez que relaciona las puntuaciones más altas con un mejor pronóstico.

Los avances logrados en el campo de la investigación neuroquirúrgica se ven reflejados en los cuidados que el profesional de enfermería implementa en las unidades de cuidados intensivos pediátricos (UCIP).

La demanda de este tipo de pacientes plantea la necesidad de la formación de un equipo interdisciplinario que aborde los cuidados y tratamientos de manera holística. De allí, la necesidad de que el enfermero cuente con competencias específicas relacionadas con las habilidades y las destrezas con una base científica necesaria para interactuar con los demás miembros del equipo, como autoliderazgo, manejo de situaciones emocionales estresantes, trabajo en equipo, comunicación efectiva, etc.

Las competencias requeridas para brindar cuidados neurocríticos constituyen un elemento clave al momento de realizar la evaluación, planificación y ejecución de un plan de cuidados oportuno y adaptado a satisfacer las necesidades del niño con lesión cerebral y su familia.

El Grupo Nacional de Enfermería Pediátrica (GRUNEP) de la SAP propone el siguiente consenso con el fin de unificar los principales cuidados, teniendo en cuenta las alteraciones de los patrones funcionales del niño con lesión cerebral, y de brindar, de esta manera, al profesional de enfermería una orientación para su manejo adecuado. 


\section{METODOLOGÍA}

Para la elaboración de esta guía, se conformó un comité integrado por profesionales del GRUNEP y del Comité Nacional de Emergencias y Cuidados Críticos de la SAP.

Se realizó una búsqueda bibliográfica en Medline/PubMed, Cochrane Library y Lilacs, TripDatabase, Cuiden, BVS, Scielo, así como en bases de datos de guías de práctica clínica. Se utilizó también bibliografía escrita recomendada por los expertos. La estrategia de búsqueda empleó los encabezados de temas médicos (Medical Subject Headings; MeSH, por sus siglas en inglés) nursing care, severe skull trauma in a Pediatric Intensive Care y sus descriptores en español cuidados de enfermería, traumatismo de cráneo grave, y abarcó publicaciones en castellano y en inglés de los últimos quince años sobre la base de la literatura especializada de la Asociación de Enfermería de América del Norte (North American Nursing Association, NANDA, 2015-2017). La búsqueda se redujo de 100 a 50 publicaciones. Se seleccionó, de acuerdo con el análisis crítico, la mejor evidencia disponible y se distribuyó entre el grupo de expertos para su análisis. La calidad de la evidencia recabada fue moderada según el sistema Grading of Recommendations, Assessment, Development and Evaluation (GRADE). ${ }^{4}$

Las recomendaciones surgidas del análisis de la evidencia científica analizada fueron sometidas a una revisión por expertos para ajustarlas a la experiencia de enfermería en la práctica asistencial, como así también desde la mirada interdisciplinaria.

\section{Objetivos según el soporte vital pediátrico avanzado ${ }^{5}$}

El programa de soporte vital avanzado propone para estos pacientes:

1. Evaluar el monitoreo del Glasgow.

2. Mantener la presión intracraneana (PIC) en valores fisiológicos.

3. Evaluar la función respiratoria.

4. Prevenir la lesión cerebral secundaria (hipoxemia e hipotensión).

5. Prevenir el shock hipovolémico manteniendo la presión sistólica $>$ del percentil 5 .

6. Mantener la normotermia del niño.

7. Aplicar medidas universales de asepsia.

8. Favorecer la mayor permanencia posible de los padres al lado del paciente.

\section{Planificación y organización de la Unidad de Cuidados Intensivos Pediátricos}

La planificación y organización constituyen las dos primeras etapas del proceso administrativo, que orientan al equipo a pensar estratégicamente qué y cómo se va a hacer para obtener un resultado eficiente y eficaz, instrumentando la utilización de los recursos humanos y materiales disponibles. ${ }^{6}$

Para ello, "es fundamental conocer, mediante una rápida anamnesis indirecta del niño lesionado, los datos más significativos del paciente que se va a recibir en la unidad de alta complejidad, entre ellos: edad, peso, Glasgow y tipo de trauma ocasionado, variables que van a permitir planificar y organizar la unidad funcional del niño/adolescente y, en consecuencia, el desarrollo del proceso de atención de Enfermería (P. A. E.)"'7 (Tabla 1).

TABLA 1. Clasificación del traumatismo encéfalo-craneano de acuerdo con la Escala de Glasgow

\begin{tabular}{lcc}
\hline Leve & Moderado & Grave \\
\hline - Glasgow 14-15 o 13-15. & - Glasgow 9-13. & - Glasgow igual a 8 o menor. \\
- Niño asintomático. & - Pérdida inicial de conciencia. & - Situación de coma. \\
- Sensorio normal. & - Déficit neurológico focal transitorio. & - Signos de fractura de cráneo \\
- Examen neurológico normal & - Hipotonía de uno o más miembros. & - Alteraciones neurológicas focales. \\
sin signos de foco. & -Vigilancia neurológica & - Vigilancia neurológica en la UCIP \\
- Sin evidencia física de fractura de cráneo. & por $12-24$ horas. & con sostén de imágenes. \\
- Vigilancia neurológica las primeras 24 horas. &
\end{tabular}

UCIP: Unidad de Cuidados Intensivos Pediátricos.

Fuente: Manual de Emergencias y Cuidados Críticos. 
Una vez realizada la evaluación, Enfermería organizará la unidad funcional con la definición concreta de los roles para evitar daños sobreagregados.

Los principales insumos que se consideran para la organización de la unidad son:

- Unidad funcional (cama de traslado), colchón térmico frío-calor, collar de Filadelfia.

- Bolsa de resucitación acorde a la edad con válvula de presión positiva al final de la espiración (positive end-expiratory pressure; $P E E P$, por sus siglas en inglés), máscara adecuada y sistema de aspiración.

- Equipo de asistencia ventilatoria mecánica (AVM), laringoscopio acorde a la edad (curvos o rectos), tubo endotraqueal (TET) acorde a la edad, cinta adhesiva o venda tipo Tensoplast, apósito hidrocoloide.

- Monitor multiparamétrico, idealmente con sensor de índice biespectral (bispectral index; $B I S$, por sus siglas en inglés), capnografía, transductor de tensión arterial invasiva, transductor para monitoreo de la PIC.

- Fluidos de reposición, drogas de intubación rápida, drogas de reanimación cardiopulmonar (RCP), drogas de sedoanalgesia, bombas de infusión.

También debe ser prioritario efectivizar el flujograma de comunicación entre los servicios de Emergencias, Neurocirugía, Traumatología, Diagnóstico por Imágenes y servicios auxiliares para facilitar acciones rápidas y oportunas. Enfermería analizará los recursos con los que cuenta (Figura 1). ${ }^{8}$

\section{Recepción del niño con traumatismo encéfalo-craneano grave en la Unidad de Cuidados Intensivos Pediátricos}

Para la recepción del niño con TEC, se recomienda considerar las siguientes acciones del equipo multidisciplinario:

- Tomar medidas de protección personal (camisolín, guantes, antiparras) previas al lavado de manos según las recomendaciones de la OMS (2009). ${ }^{9}$

- Verificar que la columna vertebral esté inmovilizada (tabla de inmovilización) y que tenga collar de Filadelfia (o, en su caso, se debe colocar) adecuado al tamaño hasta descartar una patología de la columna vertebral y / o de la médula espinal, como lesión espinal sin anormalidad radiológica conocida (Spinal Cord Injury Without Radiographic Abnormality; SCIWORA, por sus siglas en inglés).

- Trasladar al niño desde la camilla a la cama con no menos de cuatro operadores, en bloque, coordinando la movilización.

- Colocar la cabecera de la cama en un ángulo de $30^{\circ}$ y en línea media a fin de evitar el colapso de la vena yugular y potenciar el aumento de la PIC.

- Evitar la movilización del niño, sobre todo, en las primeras horas de su ingreso a la unidad de alta complejidad.

- Usar un colchón antiescara para evitar alteraciones de la integridad de la piel.

- Cotejar los datos personales del niño en el brazalete identificatorio y evaluar si es alérgico.

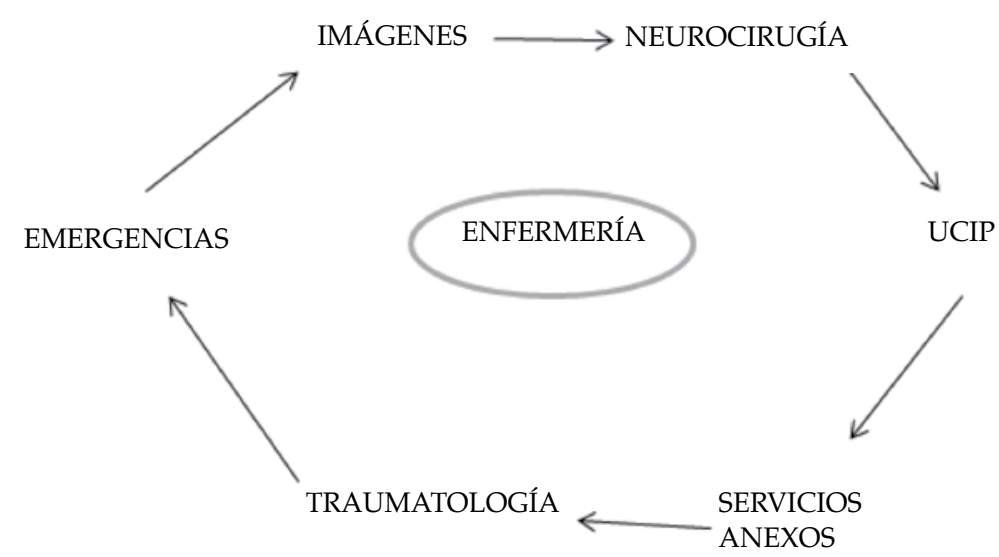

UCIP: Unidad de Cuidados Intensivos Pediátricos.

Fuente: GRUNEP, 2018. 
- Elevar las barandas de la cama del niño para prevenir caídas como parámetro de seguridad del paciente.

\section{Gestión del cuidado de acuerdo con los diagnósticos de enfermería, objetivos y acciones fundadas según patrones}

A continuación, se describe el P.A.E., compuesto por los diagnósticos según las taxonomías de la NANDA (2015-2017), con sus consecuentes objetivos, acciones de enfermería y recomendaciones.

\section{a. Patrón neurológico}

- Diagnóstico de enfermería: “Alteración en la perfusión del tejido encefálico relacionado con el aumento de la presión intracraneal secundario a la pérdida de autorregulación del flujo sanguíneo y presencia de edema cerebral".

- Objetivo: Prevenir la hipertensión endocraneana y evitar las lesiones secundarias del sistema nervioso central.

- Acciones de enfermería:

- Evaluar el estado neurológico con la GCS, considerar la intubación con valores menores de 8 o deterioro de 3 puntos.

- Evaluar el diámetro, la simetría y la reactividad pupilar dentro de cada control horario. Se considerarán asimétricas cuando su diámetro se diferencie en más de $1 \mathrm{~mm}$ y arreactivas si no se contraen menos de $1 \mathrm{~mm}$ tras iluminarlas con una luz potente. Una pupila fija dilatada indica herniación, mientras que, si son las dos, indica lesión en el tronco cerebral. Sin embargo, la hipoxemia, hipotensión e hipotermia pueden asociarse con pupilas dilatadas y con reactividad anormal (véase la Figura 2). ${ }^{10}$

- Optimizar la sedoanalgesia del niño y el bloqueo neuromuscular si procede según indicación médica (S. I. M.), utilizando instrumentos, tales como las Escalas de Ramsay y la de Confort (esta última es la más utilizada internacionalmente en pediatría). ${ }^{11}$

- Evaluar el nivel de conciencia y el estado de sedación a través del monitoreo del BIS, recordando que el rango de normalidad para la población infantil se encuentra entre 40 y 60 (véase el Anexo 2). ${ }^{12}$

- Posicionar al niño en línea media y a $30^{\circ}$ con el fin de favorecer el drenaje yugular y la disminución de la PIC.

- Utilizar un catéter para monitoreo de PIC (puede ser intraventricular o intraparenquimatoso, entre otros) en pacientes con Glasgow menor de 8 que requieren intubación o con riesgo de edema cerebral $^{13}$ (véase la Tabla 2).

FigURA 2. Evaluación pupilar

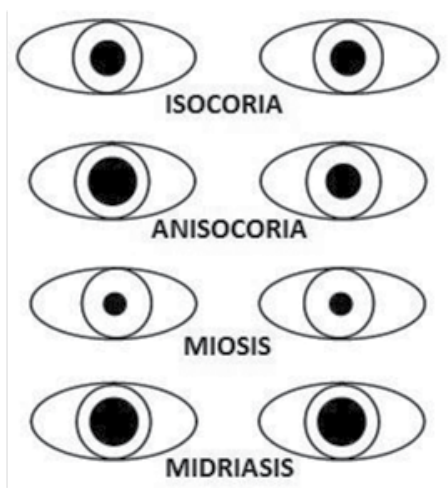

Fuente: Manual de Emergencias y Cuidados Críticos.

TABLA 2. Diferencias entre los catéteres intraventricular e intraparenquimatoso

\begin{tabular}{|c|c|}
\hline CIV & CIP \\
\hline Se coloca en quirófano; se considera el gold standard. & Puede colocarse en la unidad del paciente. Es costoso. \\
\hline $\begin{array}{l}\text { Función: 1. permite la medición de la PIC; } \\
\text { 2. permite adecuar la terapéutica mediante } \\
\text { la extracción del LCR. }\end{array}$ & $\begin{array}{l}\text { Función: medición de la PIC } \\
\text { y de la temperatura cerebral. }\end{array}$ \\
\hline $\begin{array}{l}\text { Medición a través de un catéter de silicona que } \\
\text { se inserta en el ventrículo y se conecta a un transductor de presión. }\end{array}$ & $\begin{array}{l}\text { Medición a través de fibra óptica colocada } \\
\text { en el parénquima cerebral. }\end{array}$ \\
\hline Se recomienda no mantener más de 5 días por riesgos de infección. ${ }^{11}$ & Posee bajas tasas de complicaciones. \\
\hline $\begin{array}{l}\text { Se recomienda recalibrar cada vez que se haga } \\
\text { un procedimiento y modifique su altura. }\end{array}$ & $\begin{array}{l}\text { Una vez inserto, no se puede calibrar in situ y } \\
\text { debe ser reemplazado. }\end{array}$ \\
\hline
\end{tabular}

CIV: catéter intraventricular; CIP: catéter intraparenquimatoso; PIC: presión intracraneana; LCR: líquido cefalorraquídeo.

Fuente: Rodríguez Boto G et. al. Madrid, España. Elsevier. 2012;30(1):16-22. 
- Colocar y calibrar el transductor de la PIC en cero. Armado: bajo técnica estéril. El cero se ubicará a nivel del foramen de Monro. Se debe recordar que se realiza poniendo el transductor al nivel topográfico entre el ángulo externo del ojo y el trago (véase el Anexo 3). ${ }^{14,15}$

Se deben considerar las ondas de PIC normal y PIC patológicas para tener en cuenta si los valores obtenidos son fidedignos.

- Preparar y administrar soluciones hipertónicas S. I. M., tales como manitol y clorurado hipertónico al $3 \%$ (véase el ítem "Recomendaciones de enfermería" más adelante).

- Mantener la normotermia del niño, evitando cuadros de hipertermia y de hipotermia. La hipotermia como tratamiento preventivo de la hipertensión intracraneana sigue siendo un recurso controversial que se asocia a un aumento de la mortalidad. ${ }^{16}$

- Reponer las pérdidas de líquido cefalorraquídeo (LCR) volumen a volumen, con solución de Ringer lactato S. I. M.; en caso de que el niño presente drenaje ventricular externo, se deben observar y registrar sus características.

- Recomendaciones de enfermería:

Es importante que el enfermero intensivista tenga en claro algunos valores de referencia en cuanto a la PIC:

- en la población pediátrica, los valores oscilan entre 3 y $7 \mathrm{mmHg}$.

- en los lactantes, se consideran valores $<6 \mathrm{mmHg}$.

Si bien no hay recomendaciones establecidas a partir de qué valor se comienza la terapéutica, se dan como opción valores de PIC > $20 \mathrm{mmHg}$.

La PPC es la diferencia que existe entre la presión arterial media y la PIC.

$P P C=$ tensión arterial media $($ TAM $)-$ PIC

Las guías de tratamiento pediátrico recomiendan mantener una $\mathrm{PPC}>40 \mathrm{mmHg}$ y

TABLA 3. Valores normales según el rango de edad

\begin{tabular}{lc}
\hline Población & Valor \\
\hline Lactantes y niños pequeños & De 40 a $50 \mathrm{mmHg}$ \\
Escolares & De 50 a $60 \mathrm{mmHg}$ \\
Adolescentes & $>60 \mathrm{mmHg}$ \\
\hline
\end{tabular}

Fuente: Manual de Emergencias y Cuidados Críticos. Fundasap. 2013;26-27:255-270. evitar PPC $<40 \mathrm{mmHg}$, ya que las perfusiones cerebrales menores de estos valores facilitan la isquemia cerebral ${ }^{17}$ (véase la Tabla 3 ).

En pacientes adultos, la sugerencia es mantener una $\mathrm{PPC} \geq 70 \mathrm{mmHg}$.

- Se debe considerar el agujero de Monro (mitad del trayecto entre el ángulo externo del ojo y el trago $)^{14}$ (véase la Figura 3).

- Se recomienda cerrar las llaves de tres vías, ante procedimientos que impliquen movilizar al paciente, como la higiene diaria (concluida la tarea, se debe calibrar el equipo).

- Si el paciente se traslada a estudios diagnósticos, como TAC o resonancia nuclear magnética (RNM), se recomienda, por un tiempo no superior a 30 minutos, cerrar la llave proximal al catéter intraventricular bajo técnica estéril y desconectar el transductor del monitoreo para el tránsito (se debe acordar esta práctica con el equipo tratante). Además, los dispositivos intraventriculares, como catéteres intraparenquimatosos, deben ser tratados como sistemas cerrados (técnica estéril).

- Se deben registrar cambios e informar signos de alarma, como el deterioro del estado del paciente, reflejados en la tríada de Cushing (hipertensión arterial, bradicardia y respiraciones irregulares).

- Se debe realizar la técnica de aspiración de secreciones del TET según la necesidad del niño, durante 10-15 segundos, con el uso previo de oxígeno al $100 \%$ durante 5 minutos.

FIgURA 3. Punto topográfico de Monro para la medición de la presión intracraneana

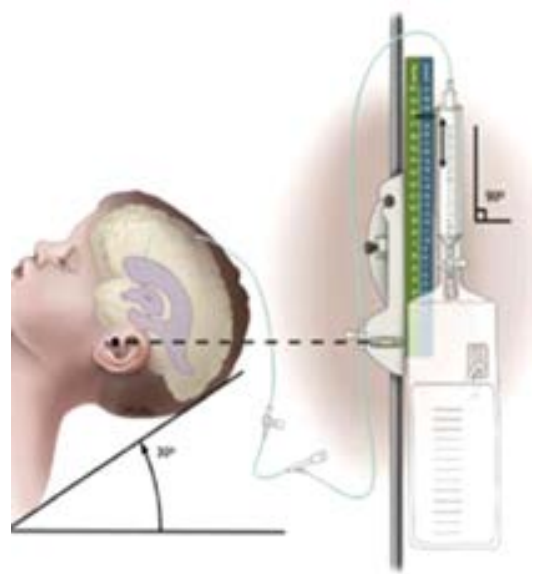

Fuente: Barrientos N. Rev. Chilena de Cirugía. 2004;56:523-527. 
El paciente se debe hallar sedado antes de los procedimientos para evitar aumentos de la PIC y las intervenciones de enfermería deben ser agrupadas en un mismo momento para evitar la lesión cerebral.

- Recomendación. La solución hipertónica cloruro de sodio al $3 \%$ se preparará de la siguiente manera: $425 \mathrm{ml}$ de agua bidestilada $+75 \mathrm{ml}$ de cloruro de sodio (ClNa) al $20 \%$ para un volumen final de $500 \mathrm{ml}$ en infusión continua con bomba de infusión y goteo S. I.M.

- Administración de difenilhidantoína (Epamin): deberá diluirse en solución fisiológica en una concentración de $6 \mathrm{mg} / \mathrm{ml}$ y administrarse por un acceso venoso solo, ya que precipita con las soluciones dextrosadas. El tiempo de infusión será de 30 minutos. ${ }^{18}$

b. Patrón hemodinámico

- Diagnóstico de enfermería: “Disminución del gasto cardíaco relacionado con alteración del volumen de eyección manifestado por disminución de la presión venosa central".

- Objetivos: Asegurar la estabilidad hemodinámica.

- Acciones de enfermería:

- Registrar los valores de la frecuencia cardíaca, presión arterial y presión venosa central (PVC), evaluando tanto la perfusión distal (pulsos periféricos y centrales) como el relleno capilar $(\mathrm{RC}<2)$.

- Controlar el ritmo diurético (RD) cada 2 horas según las necesidades del niño (valor normal -VN-: 1-2 ml $/ \mathrm{kg} / \mathrm{h}$ ).

- Nota: La hipotensión y la mala perfusión tienen efectos deletéreos en el cerebro lesionado. El percentil 5 de tensiòn arterial sistólica (TAS) puede calcularse con la siguiente fórmula:

$70 \mathrm{mmHg}+(2 \mathrm{x}$ edad en años)

A modo de ejemplo, se considera un caso.

En un niño de 5 años, la TAS será $70+$ $(2 \times 5)=$ de $80 \mathrm{mmHg}$.

- Preparar el circuito arterial, recordando que el monitoreo arterial invasivo forma parte de la evaluación del estado hemodinámico del niño (véase el Anexo 4).

- Recomendaciones de enfermería:

- Administrar los cristaloides por una vía periférica de gran calibre, ej.: basílica, cefálica, yugular externa.

- Identificar los lúmenes del catéter venoso central para organizar el trabajo. Por el lumen distal, conectar la PVC para su monitoreo, plan hídrico parenteral (PHP), correcciones y medicación. Por el lumen medial, conectar inotrópicos y sedación. Por el lumen proximal, podría usarse la nutrición parenteral (NPT) o la infusión específica de ciertas drogas depresoras, como tiopental.

- Si el niño presenta alteraciones hemodinámicas secundarias a la lesión del trauma (hipotensión), el enfermero deberá considerar la disponibilidad de cristaloides (solución fisiológica) y / o la preparación de drogas vasoactivas según el peso y S. I. M.

\section{c. Patrón respiratorio}

- Diagnóstico de enfermería: "Patrón respiratorio ineficaz relacionado con disfunción neuromuscular de los músculos respiratorios manifestado por disminución de la capacidad vital".

- Objetivos: Mantener una buena oxigenación del niño.

- Acciones de enfermería:

- Correlacionar la mecánica respiratoria con la saturometría, capnografía y la gasometría arterial (estado ácido base) (véase la Tabla 4). ${ }^{17}$

- Considerar, en todo paciente ventilado, los valores de protección pulmonar, fracción inspirada de oxígeno $\left(\mathrm{FIO}_{2}\right)$ menor del $60 \%$, volumen tidal entre 6 y $8 \mathrm{ml} / \mathrm{k}$ y presión plateau menor de 30. Los valores de saturometría recomendados serán por encima del $94 \%$ considerando que la hiperoxia incrementa la mortalidad. ${ }^{19}$

- Implementar acciones preventivas de neumonías asociadas a AVM: posición de la cabecera de la cama de 30 a 40 grados, higiene oral frecuente con colutorios antisépticos y, si correspondiera, colocar aspiración subglótica.

- Realizar fijación del TET adecuada a fin de evitar la extubación no programada del niño.

TABLA 4. Valores normales del estado ácido base

PH: 7,35/7,45

PaCO2: $35-45$ mmHg

PaO2: 80-100 mmHg

Saturación: > $94 \%$

HCO3: 22-26 meq/1

$\mathrm{PaCO}_{2}$ : presión parcial de dióxido de carbono;

$\mathrm{PaO}_{2}$ : presión parcial de oxígeno; $\mathrm{HCO}_{3}$ : bicarbonato.

Fuente: Manual de Emergencias y Cuidados Críticos. 
- Verificar la correcta ubicación del TET a través de la técnica de auscultación de ambos campos pulmonares, observar la expansión torácica, capnografía y cotejar con una placa de tórax.

- Recomendaciones de enfermería:

- Mantener la vía aérea libre de secreciones. Considerar, antes de aspirar, la administración de lidocaína al 1\% (1mg/ $\mathrm{kg} /$ dosis) para evitar el incremento de la PIC y generar un efecto protector atenuando una respuesta hemodinámica.

- La fórmula para el cálculo del número de TET como para la profundidad es la siguiente según la SAP:

$\mathrm{N}^{\circ}$ de tubo $=\frac{\text { edad en años }+4}{4}$

$\mathrm{N}^{\circ}$ de tubo con balón $\frac{=\text { edad en años }+3,5}{4}$

Profundidad: $\mathrm{n}^{\circ}$ de TET $\times 3$

\section{d. Patrón urinario}

- Diagnóstico de enfermería: “Alteración de la eliminación por defecto o exceso relacionado con trastornos de la hormona antidiurética manifestado por poliuria o oliguria".

- Objetivos: Mantener un estado de eliminación renal adecuado.

- Acciones de enfermería:

- Colocar la sonda vesical con técnica estéril, debido a que el niño se encontrará bajo efecto de la sedoanalgesia y uso de bloqueadores neuromusculares que producen la disminución de la tonicidad y se genera el globo vesical (se debe evaluar esta práctica en caso de trauma en el periné).

- Realizar un estricto balance horario de ingresos y egresos.

- Calcular el RD horario con el fin de evaluar la perfusión renal (VN: de 1 a $2 \mathrm{ml} / \mathrm{kg} /$ hora).

- Evaluar la cantidad y la calidad de la diuresis (se debenutilizar tiras reactivas urinarias para determinar el PH urinario y la densidad, con el fin de detectar alteraciones de la hormona antidiurética).

- Recomendaciones de enfermería:

- Detectar cambios en el RD debido a alteraciones en la secreción de hormona antidiurética (diabetes insípida, síndrome inadecuado de la hormona antidiurética y síndrome perdedor de sal). Se deben comunicar al equipo tratante. e. Patrón metabólico y nutricional

- Diagnóstico de enfermería: “Alteración de la nutrición por defecto relacionado con una ingesta menor y aumento del catabolismo".

- Objetivos: Mantener un estado nutricional adecuado.

- Acciones de enfermería:

- Administrar alimentación por vía enteral o parenteral según los requerimientos del niño (se debe recordar mantener el trofismo).

- La medición del residuo gástrico está desaconsejada según las guías de la Sociedad Americana de Nutrición Enteral y Parenteral (American Society for Parenteral and Enteral Nutrition, ASPEN). ${ }^{20}$

- Control de glucemia cada 6 horas o una vez por turno.

- Si la alimentación es enteral por sonda nasogástrica (SNG) o sonda orogástrica (SOG): se debe evaluar la presencia de peristaltismo, distensión abdominal, diarrea y vómito una vez por turno.

- Verificar la temperatura y la velocidad de infusión de la alimentación.

- Realizar la administración de protección gástrica para prevenir las úlceras por estrés S.I.M.

- Recomendaciones de enfermería:

- Los niños con TECG presentan hipermetabolismo, gasto energético alto y aumento de la pérdida de proteínas. El apoyo nutricional precoz previene la disminución de la inmunidad, la translocación bacteriana y la disminución de la estadía hospitalaria por consecuencia.

\section{f. Higiene, confort y seguridad del niño}

- Diagnóstico de enfermería: "Riesgo de infección relacionada con alteración de las barreras naturales del organismo".

- Objetivo: Evitar potenciales infecciones sobreagregadas.

- Acciones de enfermería:

- Disminuir el riesgo de infecciones intrahospitalarias.

- Realizar higiene bucal una vez por turno con clorhexidina al 0,12\%.

- Efectuar el baño con dos operadores, una vez por día, realizando movimientos en bloque, protegiendo la lesión cervical con el uso del collar de Filadelfia.

- Colocar protección en las zonas de apoyo del niño (maléolos, omóplatos, zona 
retroauricular, área occipital, trocánteres), uso de apósitos hidrocoloides, aerosoles siliconados y cremas para evitar lesiones en la piel.

- Recomendaciones de enfermería:

- Aprobar la participación de la familia en los cuidados básicos del niño (baño, cambio de pañal, humectación de la piel, etc.) si el estado clínico del niño lo permite.

- Utilizar la escala para medir el riesgo de úlceras por presión (UPP) mediante instrumentos validados, como el Branden $Q$ modificado, que permite clasificar el grado de riesgo con variables, tales como movilidad del paciente, actividad, sensorio, humedad, fricción, cizalla, nutrición, perfusión y oxigenación (véase el Anexo 5).

- Realizar protección ocular con lágrimas artificiales, previa higiene con solución fisiológica cada dos a cuatro horas teniendo especial cuidado en que los ojos queden bien ocluidos, con el fin de evitar potenciales úlceras corneales.

\section{Cuidado humanizado en la \\ Unidad de Cuidados Intensivos Pediátricos}

Cuidados humanizados es un término que ha comenzado a tomar relevancia global en los últimos tiempos. Actualmente, en las UCI tanto de adultos como de pediatría, el equipo de salud está tan conectado con la tecnología y los tratamientos de vanguardia que pareciera no haber un tiempo para parar y mirar al otro y reflexionar sobre qué es lo que verdaderamente está necesitando y qué aspectos se están dejando de lado. En este contexto, surge el concepto de humanización de los cuidados dentro de las UCI.

"No debemos perder de vista las heridas emocionales de los pacientes, preguntarnos qué pasa con la angustia de la familia y también con el sufrimiento del profesional".

"Tenemos que entender que los cuerpos duelen pero las personas sufrimos". Existe un llamado a los equipos de salud a reflexionar y pensar que se debe asumir el compromiso necesario para generar estrategias que favorezcan un ámbito donde la familia sea parte del cuidado, optimizando el tiempo de permanencia de los padres junto a su hijo. ${ }^{21}$

Diversas recomendaciones internacionales consideran que las visitas familiares no deben tener restricciones, aunque, lógicamente, estas deben adaptarse a los deseos del paciente y a sus condiciones clínicas. ${ }^{22}$ Nuestros pacientes tienen el derecho a sentir el afecto y el cuidado de sus familias, y, por lo tanto, se alienta el desafío de las UCI de puertas abiertas que contempla amplios horarios de permanencia junto a su ser querido.

"El cuidado humanizado dentro de la Terapia Intensiva conlleva un enfoque bidireccional"; por un lado, no hay duda de que el centro de nuestro quehacer profesional es el paciente y la unidad familiar, y hacia ellos deben ir dirigidos todos nuestros esfuerzos para garantizar no solo una correcta atención técnica, sino humana. Pero, por otro, es cierto que hay que "cuidar a los que cuidan", y que las exigencias, aun normales, en el cuidado de los pacientes graves con enfermedades agudas suelen provocar agotamiento y frustración dentro del equipo de salud (síndrome de burnout).

La enfermedad despersonaliza: el enfermo es una patología o un número de cama o una historia clínica. Pero también despersonaliza al equipo de salud: parecería existir, en el ideario colectivo, una relación inversamente profesional, en la que a mejor atención técnica (entendida como sinónimo de profesionalidad y destreza), menor cuidado humano (entendido como "flaqueza" o "sensiblería").

La vida del afuera de la Terapia Intensiva infantil no se detiene por la enfermedad. Sin embargo, tanto el paciente como su familia parecen quedar en "modo suspensión", en un limbo, yendo y viniendo permanentemente por un camino sinuoso, escabroso, inhóspito y desconocido, recibiendo indicaciones que, muchas veces, no comprenden $\mathrm{y}$, la mayoría de las veces, ni siquiera pidieron..$^{23}$

Los derechos del niño hospitalizado no son, en su esencia, diferentes de los derechos de cualquier niño, pero hay que considerar que su contenido es redefinido por la propia enfermedad. Más allá de la limitación de su intimidad y privacidad, la posibilidad del juego, la educación y la interacción con sus afectos, además de sus gustos personales (comidas, dibujos, videojuegos, vestimenta), sufren graves alteraciones que hay que tener en cuenta.

La ética del cuidado constituye no solo el cumplimiento abstracto de responsabilidades profesionales, sino que considera un enfoque centrado en la preservación de los vínculos y el fortalecimiento de un cuidado humano dentro de la UCIP.

Es así como la entrada en vigencia del nuevo Código Civil y Comercial de la Nación Argentina abrió una nueva concepción en materia de capacidad de derecho en prácticas 
médicas para la población infantil. Estos cambios sustanciales en el plexo normativo mantienen una analogía con los tratados internacionales, como es la Convención sobre los Derechos del Niño ratificados por nuestro Estado mediante la Ley de Protección Integral de los Niños, Niñas y Adolescentes, entre otras. ${ }^{24}$

La principal consecuencia de este cambio paradigmático consistió en la transición de un régimen legal rígido en materia decisoria de salud hacia uno más flexible y respetuoso de la personalidad del niño/niña y adolescente considerando su interés superior de acuerdo con su capacidad progresiva. ${ }^{25}$

\section{DISCUSIÓN}

Para la elaboración de este consenso, se ha decidido considerar la mejor evidencia disponible, revisada por expertos locales en distintas disciplinas y complementada en aquellos casos en los que existió controversia o insuficiente evidencia con su experiencia hasta tanto se dispusiera de información adecuadamente sustentada. Por lo tanto, el profesional de enfermería o interviniente en el cuidado del niño con lesión cerebral puede utilizar criterios distintos a los de este consenso para la aplicación de intervenciones, planificación y organización de cuidados diferenciales de acuerdo con su realidad institucional.

\section{CONCLUSIONES}

Las preocupaciones acerca del manejo del niño con TECG son motivo de consulta permanente entre los profesionales de enfermería que se desarrollan dentro de las unidades pediátricas de alta complejidad. Se sabe que el abordaje de un niño críticamente enfermo requiere de una planificación de cuidados diferenciales dirigidos a satisfacer las necesidades de los principales patrones afectados, dando importancia a la intervención de la familia como principal sostén.

El cuidado de estos pacientes se basa, sobre todo, en prevenir la lesión secundaria y tratar las complicaciones durante la evolución de la enfermedad.

Un equipo multidisciplinario que trabaje conjuntamente con objetivos claros, protocolos establecidos y consensuados será la clave para asegurar una atención eficiente y de calidad.

\section{REFERENCIAS}

1. Bell M, Wainwright M. Pediatric Neurocritical Care. In Fuhrman B, Zimmerman J, Clark R, Relvas M, et al (eds.). Pediatric Critical Care. 5th ed. Philadelphia: Elsevier; 2017. Págs.877-82.
2. Faul $\mathrm{M}, \mathrm{Xu} \mathrm{L}$, Wald $\mathrm{M}$, Coronado V. Traumatic brain injury in the United States: Emergency Departments, visits, hospitalisations and deaths 2002-2006. Atlanta: Centers for Diseases Control and Prevention; 2010. [Consulta: 13 de marzo de 2019]. Disponible en: https://www.cdc.gov/ traumaticbraininjury/pdf/blue_book.pdf.

3. Manrique Martínez I, Alcalá Minagorre P. Manejo del traumatismo craneal pediátrico. Protocolos diagnóstico terapéuticos de Urgencias Pediátricas SEUP-AEP. 2010. Págs.211-30.

4. Sanabria AJ, Rigau D, Rotaeche R, Selva a, et al. Sistema Grade. Metodología para la realización de recomendaciones para la práctica clínica. Aten Prim (Barc., Internet). 2013; 47(1):48-55.

5. American Heart Association. Guidelines update for CPR and ECC. 2015. [Consulta: 31 de enero de 2019]. Disponible en: https: / / eccguidelines.heart.org/wp-content / uploads / 2015/10/2015-AHA-Guidelines-HighlightsEnglish.pdf.

6. Paoloni N, Álvarez Gelves D. El proceso Administrativo. El “quid” de la administración. La Plata: Haber; 2011.

7. Palacio JM. Cuidados Neurocríticos en una unidad de alta complejidad UCIP. Med Infant. 2011; 18(3):260-4.

8. Debaisi G, Capra D, Iolster T, Landry L, et al. Normas de Categorización, Organización y Funcionamiento de las Unidades de Cuidados Intensivos e Intermedios. Med Intensiva. 2014; 31(Supl 1):2-19.

9. Organización Mundial de la Salud. Guía de la OMS sobre Higiene de Manos en la Atención de la Salud. Washington DC, 2009. [Consulta: 20 de mayo de 2018]. Disponible en: http:/ / cmas.siu.buap.mx/portal_pprd/work/sites/hup/ resources/LocalContent/247/2/guia_lavado_de_manos. pdf.

10. Hualde G, González P. Traumatismo de cráneo leve. En Vasallo J, Rufach D (eds.). Manual de Emergencias y Cuidados Críticos en Pediatría. 2. ${ }^{\text {da }}$ ed. Buenos Aires: FUNDASAP; 2013.Págs.255-61.

11. MencíaS,MarañónG.Monitorización deanalgesia, sedación y BNM en UCIP Protocolo del Grupo de Sedoanalgesia de la SECIP. [Consulta: 31 de enero de 2019]. Disponible en: http:/ / secip.com/wp-content/uploads/2018/06/Escalasde-Sedoanalgesia-Recomendadas.pdf.

12. Rodríguez Nunes R, Miranda Chaves I, García de Alencar J, Benevides Franco S, et al. Índice Bispectral y Otros Parámetros Procesados del Electroencefalograma: una Actualización. Rev Bras Anestesiol. 2012; 62(1):105-17.

13. Rodríguez Boto G, Rivero Garvía M, Gutiérrez González R, Márquez Rivas J. Conceptos básicos sobre la fisiopatología cerebral y la monitorización de la presión intracraneal. Neurología. 2015; 30(1):16-22.

14. Barrientos DN. Monitoreo de presión intracraneana: indicaciones y técnica. Rev Chil Cir. 2004; 56(6): 523-7.

15. Oddo M, Le Roux P. What are etiology, pathogenesis, and pathophysiology of elevates intracranial pressure? In: Deutschman C, Neligan P. Evidence-based practice of critical care. Philadelphia: Saunders-Elsevier; 2010.Págs.399-405.

16. Tasker RC, Akhondi-Asl A. Updating Evidence for Using Therapeutic Hypothermia in Pediatric Severe Traumatic Brain Injury. Crit Care Med. 2017; 45(10):e1091.

17. CastellanoS, RodríguezE. Traumatismode cráneograve.En Vasallo J, Rufach D (eds.). Manual de Emergencias y Cuidados Críticos en Pediatría. 2. ${ }^{\text {da }}$ ed. Buenos Aires: FUNDASAP; 2013.Págs.261-70.

18. Sberna N, Schiavi S. Difenilhidantoina. En Formulario farmacoterapéutico. 2. da ed. Buenos Aires: Fundación Garrahan; 2016.Págs.79-80.

19. Raman S, Prince NJ, Hoskote A, Ray S, et al. Admission $\mathrm{PaO}_{2}$ and mortality in critically III Children: A cohort 
Study and Systematic Review. Pediatr Crit Care Med. 2016; 17(10):e444-50.

20. McClave S, Taylor BE, Martindale RG, Warren MM, et al. Guidelines for the Provision and Assessment of Nutrition Support Therapy in the Adult Critically Ill Patient: Society of Critical Care Medicine (SCCM) and American Society for Parenteral and Enteral Nutrition (A.S.P.E.N.). JPEN J Parenter Enteral Nutr. 2016; 40(2):159-211.

21. Heras la Calle G, Martin MC, Nin N. Buscando humanizar los cuidados intensivos. Rev Bras Ter Intensiva. 2017;29(1):913.
22. Escudero D, Martín L, Viña L, Quindós B, et al. Política de visitas, diseño y confortabilidad en las unidades de cuidados intensivos españolas. Rev Calid Asist. 2015; 30(5):243-250.

23. Ciruzzi S. La limitación del soporte vital frente al Derecho Penal. En Tarrío M. Debates en torno al Derecho Penal. Buenos Aires: Cáthedra Jurídica; 2007.Págs.15-25.

24. Ley 26.061. Protección Integral delos Derechos delas Niñas, Niños y Adolescentes. InfoLEG. Buenos Aires, Argentina, 28 de septiembre de 2005.

25. Calvo Costa C. Sección 2. art. 25-26. En Código Civil y Comercial de la Nación. Anotado con la Relevancia del Cambio. Buenos Aires: Abeledo Perrot; 2015.Págs.13-28. 


\section{ANEXO 1. \\ ESCALA DE GLASGOW}

\section{Apertura ocular}

0-1 año

4. Espontánea

3. Tras estímulos verbales

2. Tras estímulos dolorosos

1. Respuesta nula

\section{1 año mayor}

4. Espontánea

3. A los gritos

2. Tras estímulos dolorosos

1. Respuesta nula

\section{Respuesta motora}

0-1

5. Loaliza el dolor

4. Retirada en flexión

3. Flexión anómala (decorticación)

2. Extensión (descerebración)

1. Respuesta nula
Mayor o = 1 año

6. Obedece ordenes

5. Localiza el dolor

4. Retirada en flexión

3. Flexión anómala (decorticación)

2. Extensión (descerebración)

1. Respuesta nula

\section{Respuesta verbal}

0-2 años

0-5 años

5. Llora normalmente, sonríe, se arrulla

4. Llora

3. Llanto/ chillido inadecuado

2. Guiñido

1. Respuesta nula
5. Palabras y frases adecuadas

4. Palabras inadeacuadas

3. Llora/ chilla

2. Gruñido

1. Respuesta nula
5 años o mayor

5. Orientado

4. Desorientado, conversa con el examinador

3. Palabras inapropiadas

2. Sonidos incompatibles 1. Respuesta nula

Fuente: Sociedad Argentina de Pediatría. Manual de Emergencias y Cuidados Críticos. 2. da edición.

Fundasap. 2013;26-27:255-270. 


\section{ANEXO 2. \\ ESCALA DE ÍNDICE BIESPECTRAL}

El índice biespectral es un parámetro del electroencefalograma que analiza el patrón de las frecuencias de las ondas cerebrales (\% de frecuencias rápidas, $\%$ de frecuencias lentas) y lo convierte en un número de "profundidad de sedación", es decir, estima el grado de actividad eléctrica cerebral, que se traduce en el nivel de conciencia.

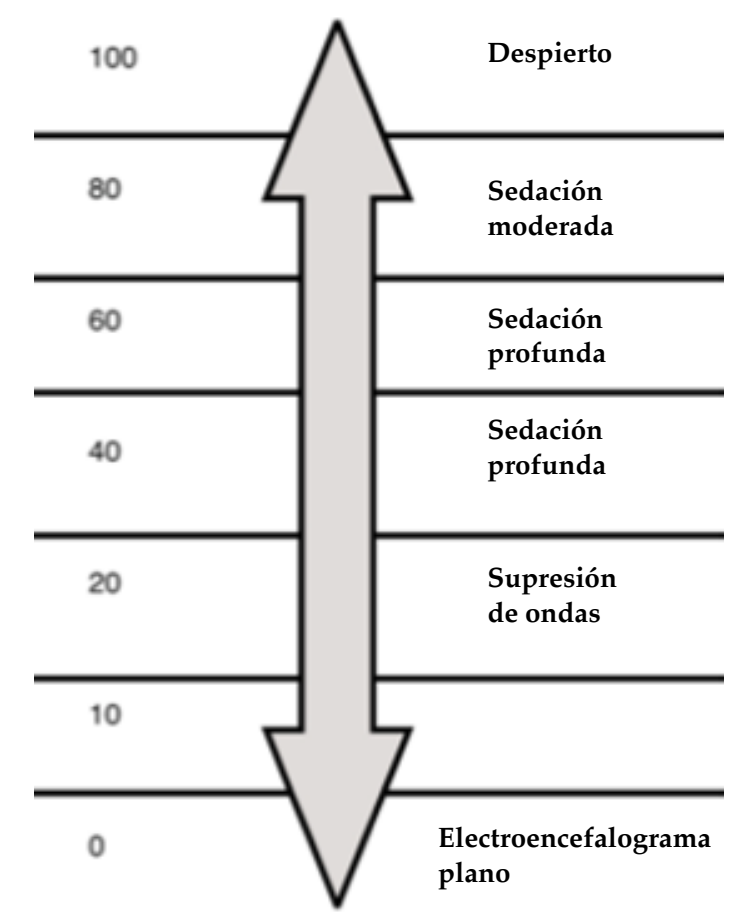

Fuente: Narbona Toledo C, Narbona Toledo F. Enferm Docente. 2010;92:10-11.

\section{Ubicación de los electrodos según la zona topográfica}

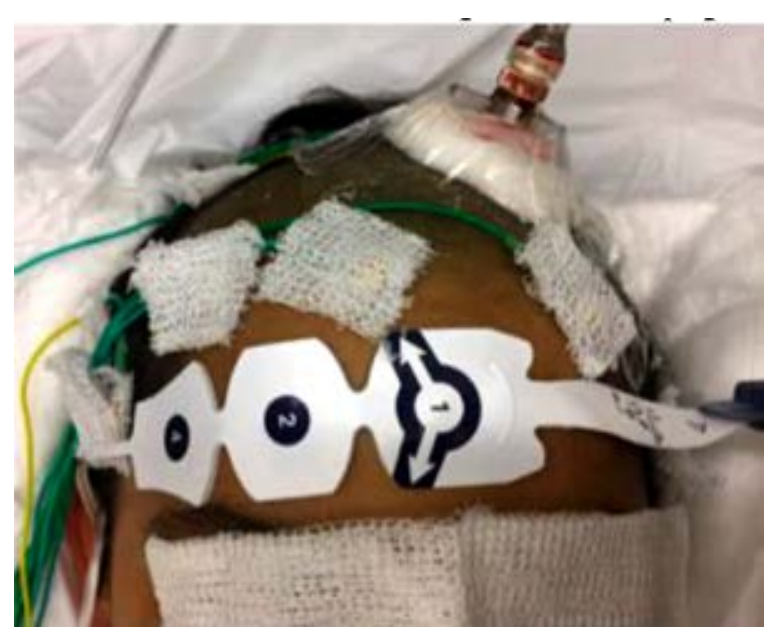

Fuente: GRUNEP, 2018.

\section{RECOMENDACIONES DE ENFERMERÍA}

Este sensor se coloca en el arco supraciliar con la limpieza previa de la piel con alcohol al $70 \%$ y consta de 4 electrodos autoadhesivos que se distribuyen según la siguiente topografía: electrodo $\mathrm{N}^{\mathrm{o}} 1$, ubicado a $5 \mathrm{~cm}$ por encima de la nariz; electrodos $\mathrm{N}^{\circ} 2$ y No 4 , ubicados por encima de la ceja; electrodo $\mathrm{N}^{\circ}$ 3, ubicado en la zona temporal derecha o izquierda según proceda. Para mayor seguridad, se recomienda presionar los electrodos durante 5 segundos para lograr una mayor adherencia. 


\section{ANEXO 3. \\ MATERIALES Y PROCEDIMIENTO DEL ARMADO DEL TRANSDUCTOR DE PRESIÓN INTRACRANEANA}

\section{MATERIALES}

1 par de guantes estériles

1 campo liso estéril

1 transductor de presión (domo)

1 llave de tres vías con tapa de seguridad

1 prolongador de $100 \mathrm{~cm}$

1 tapa de seguridad

1 jeringa de $20 \mathrm{~cm}$

2 ampollas de solución fisiológica

\section{ELEMENTOS PARA EL ARMADO DEL TRANSDUCTOR}

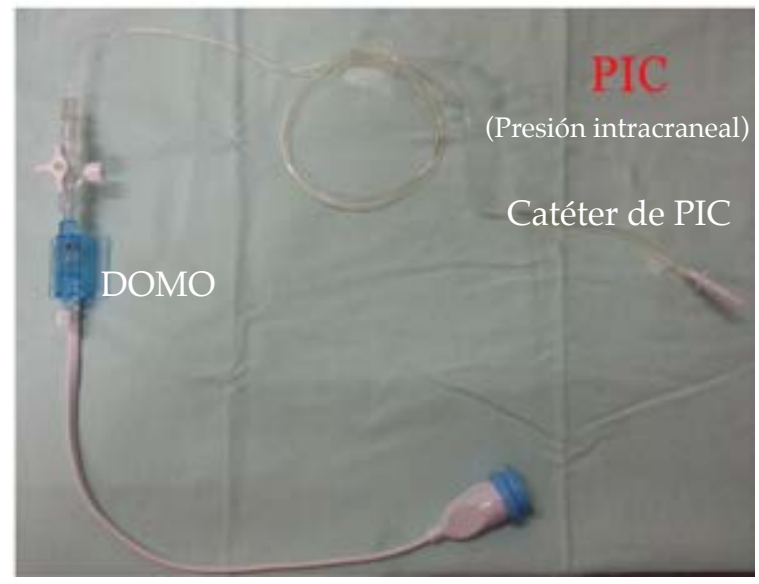

Fuente: GRUNEP, 2018.

\section{PROCEDIMIENTO:}

- Lavarse las manos según las recomendaciones del Servicio de Epidemiología e Infectología de cada institución, considerando los 5 momentos definidos por la OMS.

- Abrir el campo estéril liso y colocar los materiales necesarios para el armado del transductor de PIC.

- Colocarse los guantes bajo técnica estéril.

- Tomar el transductor y conectar, en su extremo superior, la llave de tres vías adaptada al prolongador de $100 \mathrm{~cm}$. En el extremo distal del transductor, conectar la tapa de seguridad.

- Cargar la jeringa con $20 \mathrm{~cm}$ de solución fisiológica y adaptarla a la llave de tres vías para realizar el purgado del transductor, evitando la formación de burbujas de aire.

- Fijar el transductor en un soporte de suero en la cabecera del niño para que esté cercano al catéter intraventricular.
- Conectar el transductor al cable de presión que deriva del monitor multiparamétrico y calibrar a cero.

- Establecer el cero con el transductor exactamente al nivel del foramen de Monro, con la posición supina del niño.

- Conectar (médico o enfermero) bajo técnica estéril el prolongador de $100 \mathrm{~cm}$ al catéter intraventricular.

Nota: La onda PIC es una onda de pulso sanguíneo cerebral que depende de la entrada y salida rítmica de la sangre a la cavidad craneal y de los componentes intracraneales. El origen es dado por la transmisión de la onda de pulso sanguíneo a los plexos coroideos y de estos a los componentes del compartimento intracraneal. Los trazados de monitoreo de la PIC dependen de las variaciones del contenido intravascular que se manifiestan por dos tipos de ondas, las correspondientes al latido cardíaco y las secundarias a las fases de la respiración pulmonar. Las ondas de pulso del LCR, llamadas ondas de Lundberg, normalmente, tienen tres o más picos identificados. 


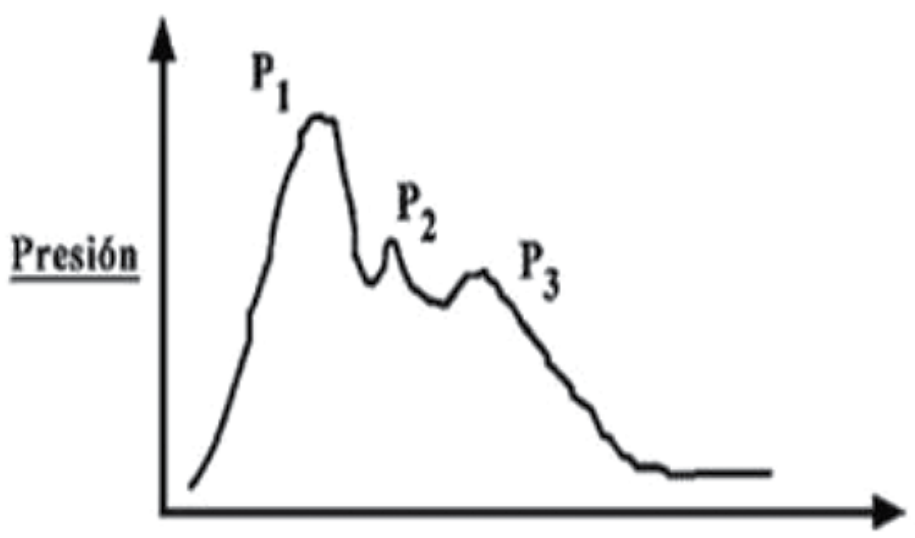

\section{Onda de presión normal Cráneo con buena distensibilidad}

\section{$\underline{\text { Tiempo }}$}

Fuente: Neligan P et ál. The Evidence Based Practice of Critical Care (2009). Elsevier. 399-405.

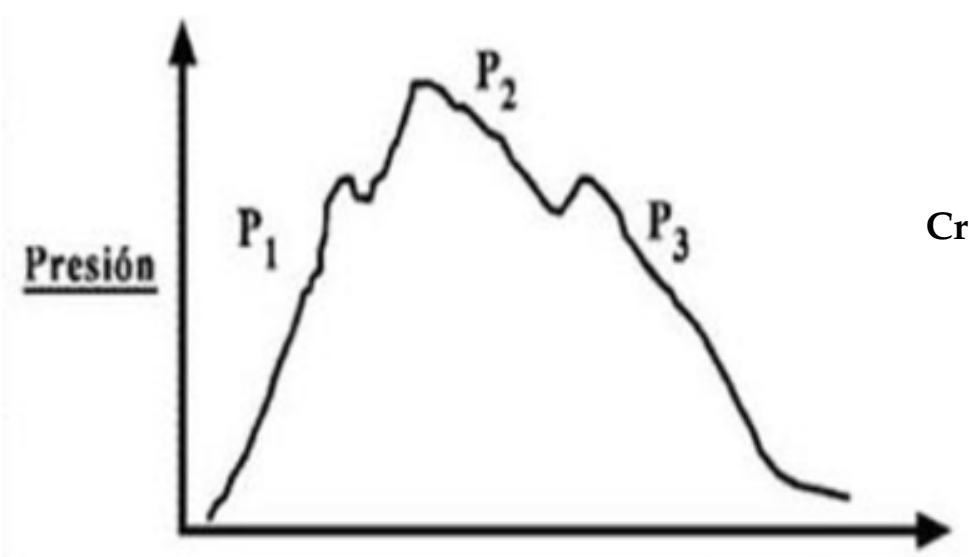

Onda de alta presión

Tiempo

\section{$P_{1}:$ Percusión (arterial) $\quad P_{2}:$ Tidal (Rebote craneal) $\quad P_{3}:$ Dicrótico (Venoso)}

$\mathrm{P}_{1}=$ Llamada onda de percusión, corresponde a la presión sistólica. Presenta un pico agudo y una amplitud consistente refleja la eyección de sangre del corazón que se transmite a través de los plexos coroideos en el ventrículo.

$\mathrm{P}_{2}=$ Llamada onda de marea, es el resultado de la presión en el LCR, tiene una amplitud y forma variable, y refleja la compliance cerebral.

$\mathrm{P}_{3}=$ Llamada onda dicrótica, refleja el cierre de la válvula aórtica, que corresponde a la presión diastólica.

Cuando la onda anteriormente descrita se modifica por condiciones de lesión cerebral, se produce una transformación morfofisiológica de la $\mathrm{P}_{2}$ la cual se observa picuda y refleja una disminución de la compliance cerebral producto de la vasodilatación y de la disminución de la PPC. 


\title{
ANEXO 4. \\ MATERIALES Y PROCEDIMIENTOS \\ DEL ARMADO DEL TRANSDUCTOR DE PRESIÓN
}

\author{
MATERIALES: \\ - Guantes y campo estéril \\ - Transductor de presión domo \\ - Intraflow (sistema de administración de flujo) \\ - Presurizador \\ - 2 llaves de tres vías con rosca de seguridad \\ - Sachet de solución fisiológica de $500 \mathrm{ml}$ con heparina de $1 \mathrm{UI} / \mathrm{ml}$ \\ - 1 sistema de perfusión macro \\ - 2 prolongadores de $150 \mathrm{~cm}$ para conectar al catéter arterial y al catéter distal del acceso central
}

\section{PROCEDIMIENTO}

- Purgar el sistema con solución fisiológica heparinizada asegurándose de que no queden burbujas en el purgado.

- Presurizar la solución heparinizada hasta $300 \mathrm{mmHg}$.

- Fijar el transductor a nivel de la línea media axilar.

- Calibrar el monitoreo a cero, considerando que la posición del niño sea supina. 


\section{ANEXO 5. \\ PROTOCOLO DE PREVENCIÓN DE ÚLCERAS POR PRESIÓN ESCALA DE BRANDEN Q MODIFICADA}

\begin{tabular}{|c|c|c|c|c|}
\hline $\begin{array}{l}\text { MOVILIDAD } \\
\text { Cambio de posición } \\
\text { de control del cuerpo } \\
\end{array}$ & $\begin{array}{l}\text { 1. Nula o no se } \\
\text { mueve sin ayuda. }\end{array}$ & $\begin{array}{l}\text { 2. Muy limitada; } \\
\text { no puede girar solo. }\end{array}$ & $\begin{array}{l}\text { 3. Limitada; puede } \\
\text { cambiar de posición solo. }\end{array}$ & 4. Sin limitación. \\
\hline $\begin{array}{l}\text { ACTIVIDAD } \\
\text { (grado de } \\
\text { actividad física) } \\
\end{array}$ & $\begin{array}{l}\text { 1. Confinada } \\
\text { a la cama. }\end{array}$ & $\begin{array}{l}\text { 2. No puede } \\
\text { tenerse en pie. }\end{array}$ & $\begin{array}{l}\text { 3. Camina ocasionalmente } \\
\text { corta distancia. }\end{array}$ & $\begin{array}{l}\text { 4. Camina fuera } \\
\text { de la UCI. }\end{array}$ \\
\hline $\begin{array}{l}\text { SENSORIO } \\
\text { (posibilidad de } \\
\text { manifestar } \\
\text { incomodidad } \\
\text { por decúbitos) }\end{array}$ & $\begin{array}{l}\text { 1. Completo: } \\
\text { no responde al dolor } \\
\text { o no puede sentirlo } \\
\text {. }\end{array}$ & $\begin{array}{l}\text { 2. Muy limitado; } \\
\text { responde solo } \\
\text { al dolor. }\end{array}$ & $\begin{array}{l}\text { 3. Algo limitado; responde } \\
\text { a órdenes verbales } \\
\text { o tiene algún trastorno } \\
\text { en la sensibilidad. }\end{array}$ & $\begin{array}{l}\text { 4. Sin compromiso } \\
\text { alguno; comunica } \\
\text { incomodidad por } \\
\text { decúbitos. }\end{array}$ \\
\hline HUMEDAD & $\begin{array}{l}\text { 1. Piel siempre } \\
\text { húmeda. }\end{array}$ & $\begin{array}{l}\text { 2. Piel frecuentemente } \\
\text { húmeda; requiere } \\
\text { el cambio de sábanas } \\
\text { cada } 8 \text { horas. }\end{array}$ & $\begin{array}{l}\text { 3. Piel ocasionalmente húmeda; } \\
\text { requiere el cambio } \\
\text { de sábanas cada } 12 \text { horas. }\end{array}$ & $\begin{array}{l}\text { 4. Piel usualmente seca; } \\
\text { requiere cambios de } \\
\text { sábanas cada } 24 \text { horas. }\end{array}$ \\
\hline $\begin{array}{l}\text { FRICCIÓN, CIZALL } \\
\text { (la piel se mueve } \\
\text { contra superficies } \\
\text { duras al correr las } \\
\text { sábanas) }\end{array}$ & $\begin{array}{l}\text { A Espasticidad, } \\
\text { contractura, prurito, } \\
\text { agitación, } \\
\text { fricción permanente. }\end{array}$ & $\begin{array}{l}\text { 2. Imposibilidad de } \\
\text { levantarse para cambiar } \\
\text { sábanas; se desliza y requiere } \\
\text { frecuentemente } \\
\text { reposicionamiento. }\end{array}$ & $\begin{array}{l}\text { 3. Se mueve libremente; } \\
\text { requiere poca } \\
\text { asistencia y pocos } \\
\text { reposicionamientos. }\end{array}$ & $\begin{array}{l}\text { 4. Se puede mover al } \\
\text { paciente completamente } \\
\text { para el cambio de } \\
\text { sábanas. }\end{array}$ \\
\hline $\begin{array}{l}\text { NUTRICIÓN } \\
\text { Ingesta habitual } \\
\text { de comida/leche }\end{array}$ & $\begin{array}{l}\text { 1. Muy pobre; ayuno o } \\
\text { líquidos claros } \mathrm{x} 5 \mathrm{días} \\
\text { o albúminas }<2,5 \mathrm{~g} / \mathrm{dl} \text { o } \\
\text { recibe menos de } \\
\text { la mitad de lo indicado. }\end{array}$ & $\begin{array}{l}\text { 2. Inadecuada. } \\
\text { NPT con aportes calóricos } \\
\text { o < que los necesarios o } \\
\text { albúmina de } 2,5-3 \mathrm{~g} / \mathrm{dl} \\
\text { o come la mitad } \\
\text { de lo indicado. }\end{array}$ & $\begin{array}{c}\text { 3. Adecuada nutrición } \\
\text { enteral o NPT con aportes } \\
\text { adecuados. Recibe más de } \\
\text { la mitad de lo indicado y, } \\
\text { a veces, } \\
\text { rehúsa una ración. }\end{array}$ & $\begin{array}{l}\text { 4. Excelente; aportes } \\
\text { adecuados para la edad. } \\
\text { Recibe todas sus } \\
\text { raciones. }\end{array}$ \\
\hline PERFUSIÓN-OXIG & $\begin{array}{l}\text { ENACIÓN } \\
\text { 1. Muy comprometido. } \\
\text { TAM }<50 \text { mmHg } \\
\text { (40 mmHg en menos } \\
\text { de } 3 \text { meses). } \\
\text { No tolera cambios } \\
\text { de posición. }\end{array}$ & $\begin{array}{c}\text { 2. Comprometidos. } \\
\text { Normotenso, sat. }<95 \% \\
\text { o Hb }<10 \mathrm{o} \\
\text { relleno capilar }>2 " \\
\text { acidosis }(\mathrm{PH}<7,36) \\
\end{array}$ & $\begin{array}{l}\text { 3. Adecuados. } \\
\text { Normotenso, sat. <95\% } \\
\text { o } \mathrm{Hb}<10 \mathrm{o} \\
\text { relleno capilar } \\
>2 \text { " }(\mathrm{PH}>7,35) .\end{array}$ & $\begin{array}{c}\text { 4. Excelente; } \\
\text { Normotenso, sat. > 95\% } \\
\text { o Hb } 10 . \\
\text { Relleno Capilar < 2". }\end{array}$ \\
\hline
\end{tabular}

UCI: Unidad de Cuidados Intensivos; NPT: nutrición parenteral; TAM: tensión arterial media. 


\section{CLASIFICACIÓN SEGÚN EL GRADO DE RIESGO}

Alto riesgo: 0-16 puntos

Moderado riesgo: $17-22$ puntos

Bajo riesgo: $>23$ puntos

\begin{tabular}{|c|c|c|c|c|}
\hline Actividad & Bajo & Moderado & Alto & Observaciones \\
\hline Examen de la piel & Diario & Diario & Por turno & $\begin{array}{l}\text { Prominencias óseas, punto de } \\
\text { apoyo, sequedad, eritema, } \\
\text { maceración, etc. }\end{array}$ \\
\hline Higiene de la piel & Diario & Diario & Diario y/o según necesidad & Paños para higiene corporal \\
\hline Cambios posturales & Cada 4 horas & Cada 2 horas & Cada 2 horas & Colocación de colchón de aire \\
\hline $\begin{array}{l}\text { Protección de } \\
\text { los talones }\end{array}$ & $\begin{array}{c}\text { Talonera } \\
\text { hidrocelular }\end{array}$ & $\begin{array}{c}\text { Talonera } \\
\text { hidrocelular }\end{array}$ & $\begin{array}{l}\text { Talonera hidrocelular } \\
\text { o hidrocoloides gruesos }\end{array}$ & \\
\hline $\begin{array}{l}\text { Medición de } \\
\text { riesgo }\end{array}$ & Cada 2 días & 1 vez al día & 1 vez por turno & $\begin{array}{l}\text { Reevaluación en caso } \\
\text { de mala evolución }\end{array}$ \\
\hline
\end{tabular}

Fuente: Curley M A et al. Pediatr Crit Care Med. 2003; 4(3):284-290.

Se debe recordar que las zonas de presión más frecuentes son occipital, pabellones auriculares, escápulas, sacras, coccígea, maleolares, talones; por eso, se recomienda poner énfasis en el cuidado de la integridad de la piel. 